\title{
Comparação do desempenho de pré-escolares, mediante teste de desenvolvimento de Denver, antes e após intervenção nutricional
}

\author{
M. Fisberg, M.R. Pedromônico, *J .A.P. Braga, **A.M.A. Ferreira, C. Pini, S.C.C. Campos, S.O. Lemes, \\ S. SILVA, R.S. SILVA, T.M. ANDRADE
}

Disciplinas de Nutrição e M etabolismo e de*Pediatria Clínica — Departamento de Pediatria, Universidade F ederal deSão Paulo-E scola Paulista de Medicina; **Secretaria de Bem Estar Social e da Família da Prefeitura de São Paulo, São Paulo, SP.

RESUMO - A análise de performance psicomotora de crianças institucionalizadas é de fundamental importância no planejamento de atividades educativas. Estudos anteriores têm mostrado prejuízos desta função em crianças de creches.

Oвj etivo. Comparar o desempenho no teste de triagem de Denver em crianças de 2 a 6 anos de idade, de creches conveniadas com a Prefeitura de São Paulo, antes e após seis meses de intervenção nutricional com suplemento alimentar enriquecido com ferro.

MÉtodo. Foram analisadas 130 crianças de 2 a 6 anos de idade, em três creches municipais de São Paulo, aplicando-se o teste de Denver, por psicólogas treinadas, comparando-se os resultados de acordo com o sexo, faixa etária e estado nutricional, antes e após período de suplementação alimentar.

\section{INTRODUÇÃO}

De um modo geral, as estatísticas de mortalidade e morbi dade, embora bastante incompletas, são unânimes em apontar o binômio desnutrição-infecção como o principal responsável pelas condições desfavoráveis de saúde da população brasileira ${ }^{1}$.

No Brasil morrem, diariamente, por desnutrição cerca de 1.000 crianças nas faixas etárias de 1 a 6 anos. Das que sobrevivem, cerca de $53 \%$ sofrem de subnutrição².

O primeiro ano de vida é o período mais crítico do desenvolvimento; o crescimento é bastante acentuado, o sistema nervoso, de um modo geral, e o cérebro, em particular, são altamente vul neráveis aos efeitos da desnutrição. A criança apresenta altas necessidades nutricionais para prover o crescimento e desenvolvimento adequados ${ }^{3}$.

Estudos levados a efeito em diferentes países da América Latina têm demonstrado que as anemias nutricionais por carência de ferro, vitamina $B_{12}$ e áci do fólico assumiram importância crescente a partir da década de $70^{4}$.
Resultados. A maior parte das crianças teve desempenho normal, tanto na primeira aplicação $(70,80 \%)$, como na segunda $(80,80 \%)$, sem modificação do estado nutricional. Na comparação dos resultados, $76,92 \%$ não modificaram o desempenho e $\mathbf{1 8 , 4 6 \%}$ melhoraram significativamente. Em relação ao sexo, não foram encontradas diferenças significantes, enquanto que, para a faixa etária, houve melhora significante entre as crianças de 4 a 6 anos.

ConClusões. Além do aspecto nutricional, fatores como prontidão para aprendizagem, organização familiar e orientação psicopedagógica das creches devem estar favorecendo o desenvolvimento, mesmo considerando-se o baixo nível socioeconômico da população estudada.

Unitermos. Desenvolvimento. Teste de Denver. Creche. Suplemento alimentar.

Aukett ${ }^{5}$, em estudo que examinou a performance de crianças em teste de desenvolvimento, após terapia com suplementação de ferro durante 2 a 3 meses, teve como resultado estabilidade dos escores no teste, embora apresentassem boa resposta hematológica à terapia.

Um fato amplamente reconhecido é que a maior parte dos problemas que afetam o crescimento e desenvolvimento da criança e, até mesmo, sua sobrevivência decorre de situação sócio-econômica desfavorável; ou seja, a desnutrição é uma doença social ${ }^{6}$.

A realidade brasileira mostra-nos que 0 atendimento às crianças, do primeiro ano de vida até a idade de seis anos, é realizado, na mai oria das vezes, por creches, que têm por objetivo proteger e propiciar cuidados integrais de higiene, alimentação, educação e saúde, em um clima afetivo, estimulante e seguro a essas crianças ${ }^{7}$.

No Brasil, as creches passaram, desde sua criação, de um caráter filantrópico de atendimento a uma preocupação educacional, buscando suprir, além das necessidades alimentares e de higiene, aspectos mais abrangentes do desenvolvimento, como socialização, autonomia e segurançå. 
Do ponto de vista psicológico, o atendimento em creche tem sido, muitas vezes, apontado como prejudicial ao desenvolvimento mental e, sobretudo, emocional da criança, por fugir ao modelo de cuidado materno afetivo, individualizado e estável, preconizado como garantia do desenvolvimento sadio, durante os primeiros meses de vida da criança?.

Por outro lado, Prugh \& $\mathrm{Harlow}^{10}$ referem que a separação, nos três primeiros meses de vida, raramente transtorna a criança, quando uma adequada mãe substituta proporciona-Ihe cuidado. Parece que, entretanto, o segundo semestre de vida é um período muito vulnerável da criança, pois, nessa idade, el a começa a se diferenciar como ser independente da mãe e a formar uma imagem corporal primitiva, apesar de ainda existirem relações de grande dependência.

Assim, buscando investigar essas relações, o presente trabalho teve por objetivo comparar o desempenho, no teste de desenvolvimento de Denver, de crianças entre 2 e 6 anos de idade que freqüentam três creches conveniadas com a Prefeitura de São Paulo, antes e após intervenção nutricional com suplemento alimentar completo.

\section{MATERIAL E MÉTODO}

Participaram deste estudo 144 crianças, entre 2 e 6 anos de idade, sendo 76 do sexo masculino e 68 do feminino. As crianças da amostra eram provenientes de três creches conveniadas com a Prefeitura Municipal de São Paulo, com atendimento em período integral, priorizando famílias de baixa renda (0 a 3 salários-mínimos), cujas mães trabalhavam fora de casa. Além disto, todas as crianças faziam parte de um programa desenvolvido pela Disciplina de Nutrição e Metabolismo da U niversidade F ederal de São Paulo. Nesse programa, a intervenção nutricional era feita com alimento protéico-calórico enriqueci do com ferro, al cançando $100 \%$ das recomendações de ferro e proteína e $25 \%$ das recomendações energéticas, para a faixa etária pré-escolar. A análise desses dados, do ponto de vista nutricional, será fruto de outra publicação.

No presente estudo, as crianças foram avaliadas por meio do teste de triagem de desenvolvimento de Denver ${ }^{11}$, por profissionais previamente treinados. A avaliação foi realizada antes e após período de seis meses da intervenção nutricional.

O teste de Denver é um teste de triagem que leva em consideração o avanço da idade e avalia quatro áreas do desenvolvimento: motor-grosso, motor adaptativo, pessoal-social e linguagem, permitindo que sejam atribuídas, de acordo com os critérios de aval iação previstos no Manual doteste ${ }^{11}$, as categorias diagnósticas - normal, não-normal, questionável e não-testável. Segundo esses critérios de avaliação, foram considerados normais aqueles protocolos nos quais ocorreu, no máximo, uma falha de execução por área investigada. O protocolo foi considerado nãonormal quando ocorreram duas ou mais falhas em, pelo menos, duas áreas, ou, ainda, uma área com uma ou mais falhas e outra área com uma falha e nenhum outro item de avaliação executado pela criança. Em situações intermediárias, foi atribuído como diagnóstico a categoria de questionável. Foram, ainda, considerados não-testáveis os protocolos em que a criança recusava executar tantos itens, que impedia a apreciação de seu desempenho em uma ou mais áreas.

O diagnóstico não-testável foi desconsiderado da análise dos resultados por não avaliar o desempenho das crianças, resultando em uma amostra final de 130 crianças, sendo 63 do sexo masculino e 67 do feminino.

Para análise dos resultados, os diagnósticos obtidos na 1a e 2 ap aplicação foram comparados, a fim de observarmos a ocorrência ou não de diferenças no desempenho das crianças.

I nicialmente, trabalhou-se com a amostra total; em seguida, consideraram os dados obtidos em cada creche. A pós essa etapa, foram consideradas as variáveis sexo e faixa etária para comparação dos diagnósticos. Em função das características da amostra, essa foi subdividida em duas faixas etárias: grupo A (2 anos $a 4$ anos e 5 meses) e grupo $B$ ( 4 anos e seis meses a 6 anos), sendo 45 crianças do grupo $A$ e 85 do B.

\section{Análise estatística}

Em virtude do nível de mensuração das variáveis envolvidas no estudo, recorreu-se à análise estatística não-paramétrica.

Por se tratar de amostras correlacionadas, nas quais existem duas medidas para um único individuo - no caso $1^{\text {a }}$ e $2^{\mathrm{a}}$ apli cação do teste - , utilizouse o teste de Kappa e o teste de McNemar ${ }^{12-13}$.

Adotou-se nível de significância 0,05 para os testes estatísticos realizados, e quando ocorreram diferenças significantes, o número foi marcado com um asterisco.

\section{RESULTADOS E DISCUSSÃO}

Os resultados foram trabalhados, comparandose os diagnósticos de primeira e segunda aplicação, em relação à amostra total e, posteriormente, por creche. Num segundo momento, esses diagnósticos foram comparados segundo sexo e faixa etária. Com relação ao sexo, não foram encontradas diferenças significantes. 


\begin{tabular}{|c|c|c|c|c|c|c|c|c|}
\hline \multirow[t]{2}{*}{ Denver 1} & \multicolumn{8}{|c|}{ Denver 2} \\
\hline & \multicolumn{2}{|c|}{ Normal } & \multicolumn{2}{|c|}{ Questionável } & \multicolumn{2}{|c|}{ Não-normal } & \multicolumn{2}{|c|}{ Total } \\
\hline Normal & $\begin{array}{l}86 \\
(66,15 \%)\end{array}$ & $93,50 \%$ & $\begin{array}{l}6 \\
(4,62 \%)\end{array}$ & $6,50 \%$ & 0 & & $\begin{array}{l}92 \\
(70,80 \%)\end{array}$ & $100,00 \%$ \\
\hline Questionável & $\begin{array}{l}17 \\
(13,08 \%)\end{array}$ & $58,60 \%$ & $\begin{array}{l}12 \\
(9,23 \%)\end{array}$ & $41,40 \%$ & 0 & & $\begin{array}{l}29 \\
(22,30 \%)\end{array}$ & $100,00 \%$ \\
\hline Não-normal & $\begin{array}{l}2 \\
(1,53 \%)\end{array}$ & $22,20 \%$ & $\begin{array}{l}5 \\
(3,85 \%)\end{array}$ & $55,60 \%$ & $\begin{array}{l}2 \\
(1,54 \%)\end{array}$ & $22,20 \%$ & $\begin{array}{l}9 \\
(6,90 \%)\end{array}$ & $100,00 \%$ \\
\hline Total & $\begin{array}{l}105 \\
(80,80 \%)\end{array}$ & & $\begin{array}{l}23 \\
(17,70 \%)\end{array}$ & & $\begin{array}{l}2 \\
(1,50 \%)\end{array}$ & & $\begin{array}{l}130 \\
(100,00 \%)\end{array}$ & \\
\hline \multicolumn{4}{|c|}{ Teste de concordância de Kappa } & \\
\hline \multirow{2}{*}{$\mathrm{K}=, 405$} & $(\mathrm{Kw})=, 459$ & \multicolumn{2}{|l|}{$\operatorname{Se}(K)=, 732$} & \multicolumn{3}{|c|}{$\begin{array}{l}\text { Teste de McNemar: } \\
\begin{array}{ll}X^{2}=10,800^{*} & X^{2}(1 \mathrm{gL}, 5 \%)=3,84\end{array}\end{array}$} & & \\
\hline & & \multicolumn{2}{|l|}{$Z=21,807$} & \multicolumn{3}{|c|}{$\begin{array}{l}\text { Concordância diagonal }=76,92 \% \\
\text { Acima da diagonal }=4,62 \% \text { (piora) }\end{array}$} & & \\
\hline
\end{tabular}

I nicialmente, os resultados mostraram tendência geral de melhora observada no desempenho normal da mai or parte das crianças $(70,80 \%)$ na 1 ạ aplicação e que, após o período de intervenção nutricional, passou para $80,80 \%$ em relação à amostra total (tabela 1).

Essa tendência foi confirmada ao observarmos que $18,46 \%$ das crianças tiveram mel hora significante no desempenho, distribuída da seguinte forma: 13,08\% das crianças com desempenho questionável passaram para normal; 3,85\% com desempenho não-normal passaram para questionável e, finalmente, 1,53\% com desempenho não-normal passaram para normal.

Ainda, segundo tabela $1,76,92 \%$ das crianças mantiveram o mesmo desempenho, distribuído da seguinte forma: $66,15 \%$ apresentaram desempenho normal, 9,23\% desempenho questionável e 1,54\% desempenho não-normal .

Esse resultado, mais do que atestar a favor da intervenção nutricional, coloca uma questão mais antiga, que é: crianças provenientes de famílias de baixa renda apresentam "déficit no desenvol vimento"? Nessa população, a maioria $(66,15 \%)$ das crianças apresentou desempenho normal , ou seja, de acordo com o avanço da idade.

$\mathrm{Na}$ tabela 2 , observamos que $73,33 \%$ das crianças obtiveram desempenho normal na 1a aplicação e que, após o período de intervenção nutricional, esse resultado não se modificou.

Na tabela 3, observamos $69,42 \%$ das crianças com desempenho normal na 1a aplicação; após o período de intervenção nutricional, esse resultado aumentou para $84,70 \%$ das crianças. Na comparação dos diagnósticos segundo faixa etária, as crianças do grupo B (4 anos e 6 meses a 6 anos) apresentaram melhora significante de desempenho (21,18\%) em relação às do grupo $A$ ( 2 anos a 4 anos e 5 meses), que apresentaram mel hora nãosignificante de $13,33 \%$, conforme análise das tabelas 2 e 3 . Assim, as crianças mais velhas tiveram melhor desempenho no teste após o período de intervenção.

Esse resultado pode estar relacionado, entre outros fatores, a uma maior preocupação psicopedagógica das creches com as crianças do grupo B e, talvez, uma mai or prontidão para aprendizagem por parte dessas crianças.

$\mathrm{Na}$ análise dos resultados por creche, as tabelas 4 e 6 mostram que, embora a mel hora de desempenho não tenha sido significante, comparativamente, $62,50 \%$ das crianças tiveram desempenho normal na crechel e 72,72\% na crechell I . Além disso, observouse ausência de diagnósticos não-normais, após o período de intervenção.

A creche II foi a única que apresentou melhora significante $(24,07 \%)$ no desempenho das crianças (tabela 5). U ma hi pótese levantada para esteresultado está relacionada a uma maior concentração de crianças da faixa etária $B$, nessa creche.

Um outro fator a ser considerado é que utilizamos, nesse estudo, um teste de triagem de desenvolvimento que não tem especificidade para avaliar nuanças qualitativas do desenvolvimento mental. Desconhecemos o quanto e como a representação de atividades como pular, jogar, desenhar, al imentar-se, interagir está sendo elaborada pela criança.

E m contrapartida, foi o instrumento utilizado nas duas avaliações, abrangendo ampla faixa etária e 


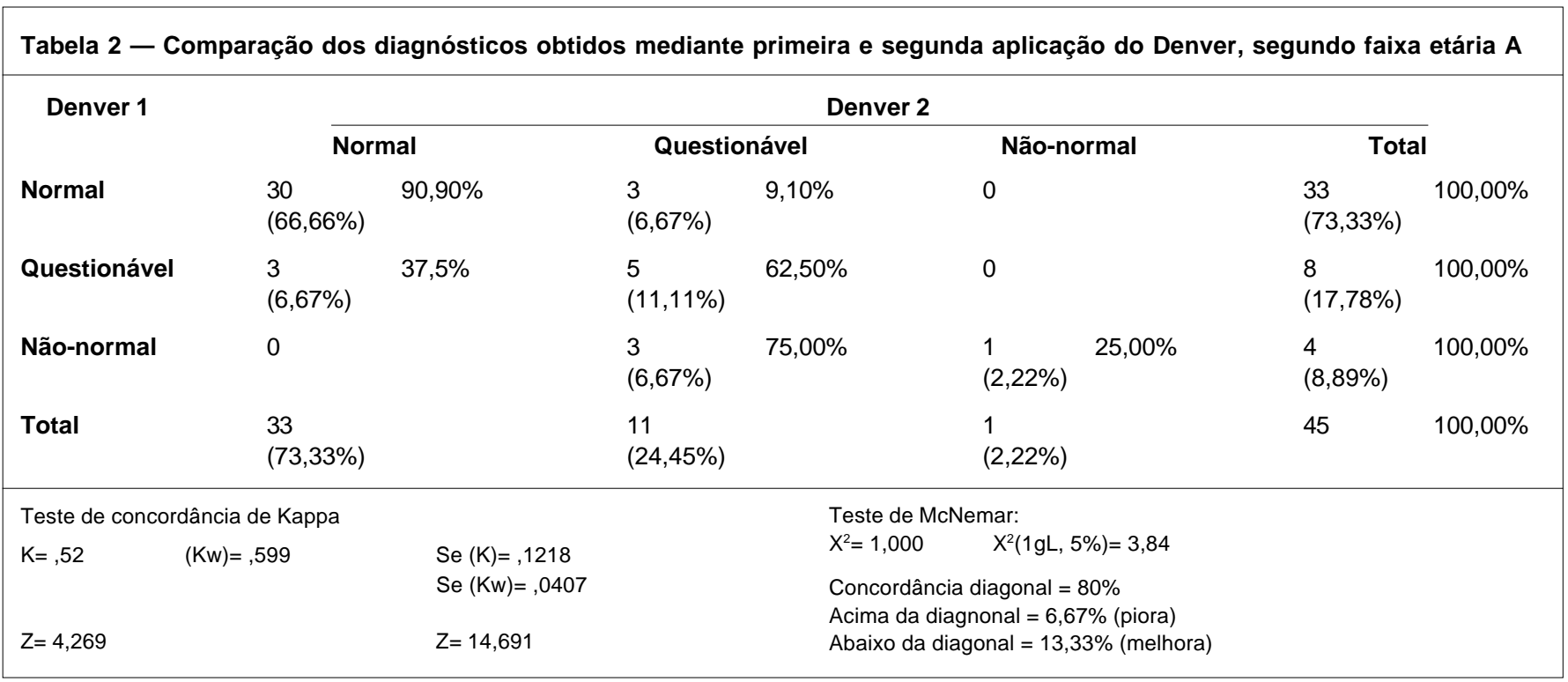

Tabela 3 - Comparação dos diagnósticos obtidos mediante primeira e segunda aplicação do Denver, segundo faixa etária B

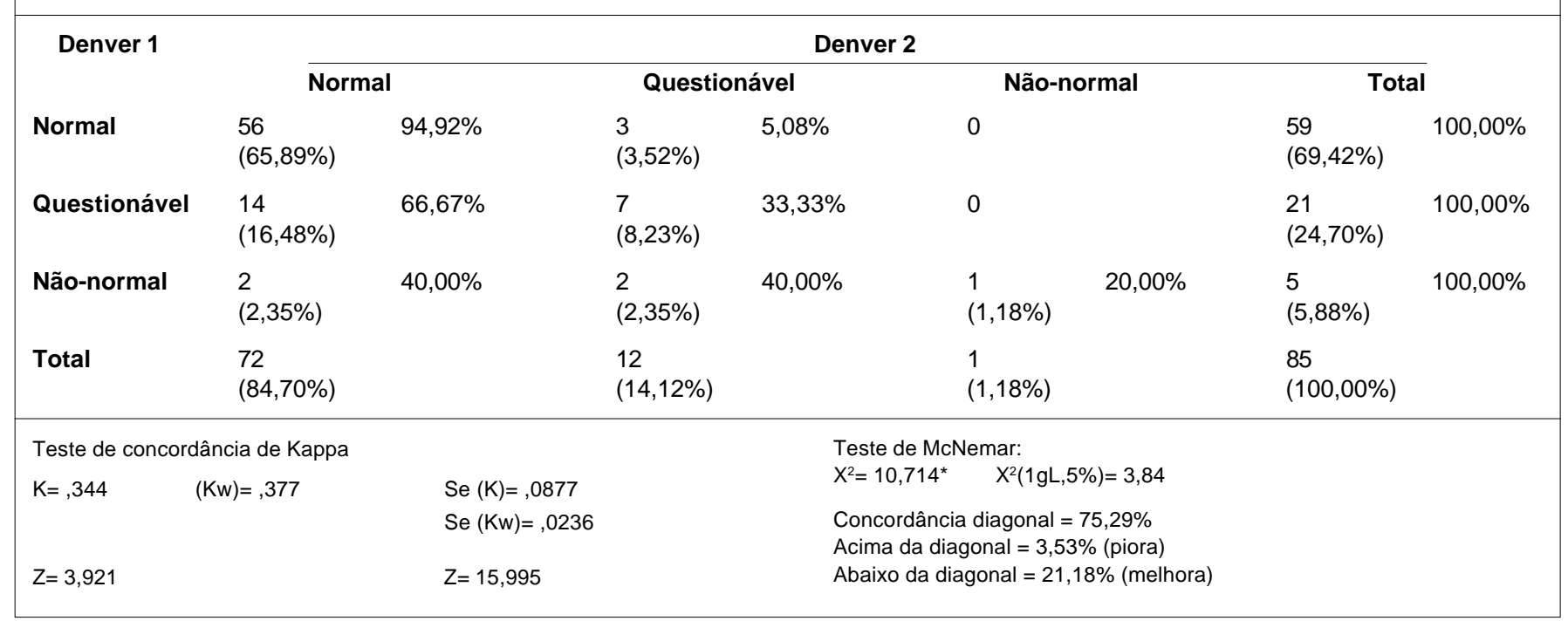

que permitiu a comparação delas. Assim sendo, os resultados permitem-nos inferir que as crianças estudadas estão aproveitando a estimulação recebida, tanto em nível nutricional quanto de vivências motoras, socialização e comunicação, de forma gl obal.

E mbora um ol har mais geral ateste a favor do desenvolvimento cognitivo, motor e emocional de crianças de 2 a 6 anos de idade provenientes de população de baixa renda, é fundamental buscarmos a superação de limites e a melhora de condições, até mesmo por considerarmos a fragilidade do instrumento utilizado.

Nesse sentido, sugerimos uma análise futura, comparando as creches em relação aos aspectos acima citados, além de investigação mais aprofundada das diferenças por faixa etária.

\section{CONCLUSÕES}

A maioria das crianças da população estudada apresentou categoria diagnóstica normal, de acordo com o teste de desenvolvimento de Denver, antes e após intervenção nutricional.

$18,46 \%$ das crianças apresentaram mel hora significante de desempenho, após o período de intervenção nutricional.

Foram encontradas diferenças significantes na comparação dos diagnósti cos na faixa etária B , ou seja, as crianças entre quatro anos e seis meses e seis anos de idade apresentaram melhora no desempenho.

As creches I elll apresentaram ausência de diagnósticos não-normais, após o período de intervenção. 


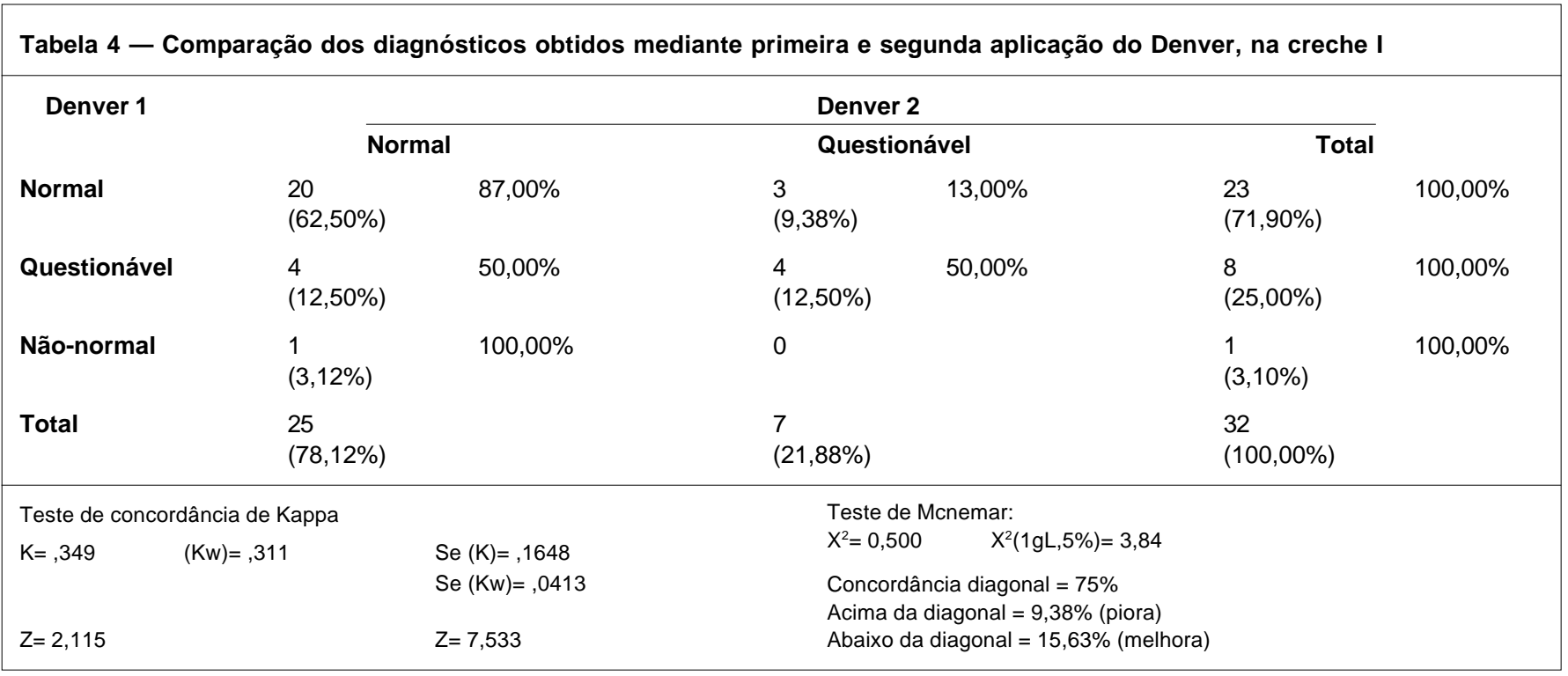

Tabela 5 - Comparação dos diagnósticos obtidos mediante primeira e segunda aplicação do Denver, na creche II

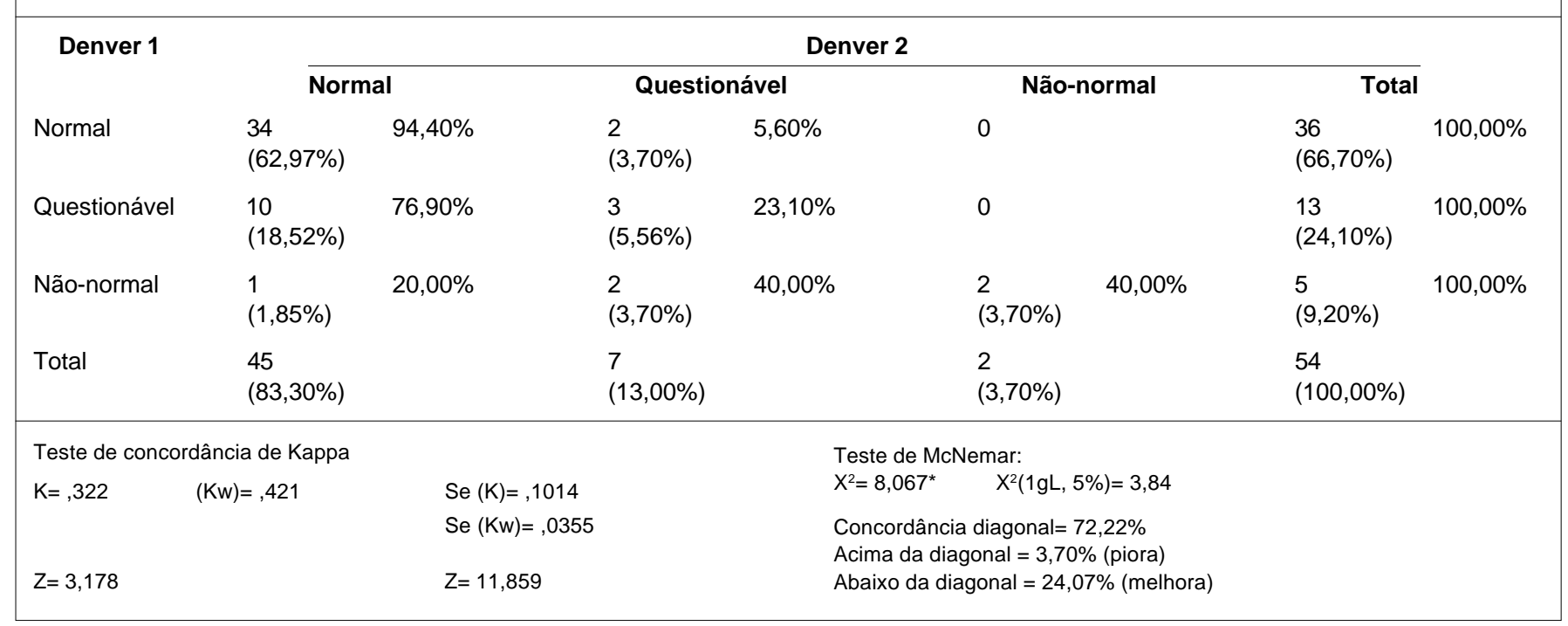

A creche II apresentou melhora significante $(24,07 \%)$ no desempenho das crianças, após o período de intervenção.

\section{SUMMARY}

Comparison of preschool children's performance through Denver developmental test, before and after nutritional supplement

Psychomotor and development analysis must be emphasized when studying institutionalized children. Many previous investigations have been showing deleterious effects of day care centers over developmental performance in children.
OBJ ECTIVE. This study is aimed at comparing the performance in the Development Screening Test (Denver) in children attending day care centers, before and after nutritional intervention with an energetic supplement enriched with iron.

METHOD. 130 children from 2 to 6 years old, attending three municipal day care centers, were evaluated by means of the application of the Denver test, by trained psychologists, comparing the collected data according to sex and agegroup, beforeand after six months intervention with iron enriched protein energetic supplement.

Results. Most of the children had normal performance, both in first application (70.80\%), and in the second one (80.80\%). When comparing these 


\begin{tabular}{|c|c|c|c|c|c|c|}
\hline \multirow[t]{2}{*}{ Denver 1} & \multicolumn{5}{|c|}{ Denver 2} & \\
\hline & \multicolumn{2}{|c|}{ Normal } & \multicolumn{2}{|c|}{ Questionável } & \multicolumn{2}{|c|}{ Total } \\
\hline Normal & $\begin{array}{l}32 \\
(72,72 \%)\end{array}$ & $\begin{array}{l}97,00 \% \\
(2,28 \%)\end{array}$ & $\begin{array}{l}1 \\
(75,00 \%)\end{array}$ & $3,00 \%$ & 33 & $100,00 \%$ \\
\hline Questionável & $\begin{array}{l}3 \\
(6,82 \%)\end{array}$ & $37,50 \%$ & $\begin{array}{l}5 \\
(11,36 \%)\end{array}$ & $62,50 \%$ & $\begin{array}{l}8 \\
(18,20 \%)\end{array}$ & $100,00 \%$ \\
\hline Não-normal & 0 & & $\begin{array}{l}3 \\
(6,82 \%)\end{array}$ & $100,00 \%$ & $\begin{array}{l}3 \\
(6,80 \%)\end{array}$ & $100,00 \%$ \\
\hline Total & $\begin{array}{l}35 \\
(79,50 \%)\end{array}$ & & $\begin{array}{l}9 \\
(20,50 \%)\end{array}$ & & $\begin{array}{l}44 \\
(100,00 \%)\end{array}$ & \\
\hline \multicolumn{3}{|c|}{ Teste de concordância de Kappa } & \multicolumn{3}{|c|}{ Teste de McNemar: } & \\
\hline \multirow[t]{2}{*}{$\mathrm{K}=5,66$} & \multirow[t]{2}{*}{$(K w)=, 622$} & \multirow{2}{*}{$\begin{array}{l}\text { Se }(K)=, 1296 \\
\text { Se }(K w)=, 0333\end{array}$} & $X^{2}=3$ & $X^{2}(1 g L$ & & \\
\hline & & & $\begin{array}{l}\text { Conc } \\
\text { Acim }\end{array}$ & $\begin{array}{l}\text { cia diagonal } \\
\text { liagonal }=2,\end{array}$ & & \\
\hline$Z=4,365$ & & $Z=18,645$ & Abai & diagonal $=1$ & & \\
\hline
\end{tabular}

results, $76.92 \%$ of the children had not altered their performance and $18.46 \%$ improved it substantially. As to sex, no significant differences werefound and as to age group, there was significant improvement among children aged 4 to 6 years of age.

Conclusions. Besides the nutritional aspects, factors such as learning readiness, family organization, and psychopedagogic orientation to the day care centers, must have fostered development, even if the low socioeconomic level of the studied population is consi dered. [Rev Ass Med Brasil 1997; 43(2): 99-104.]

KEY WORDS: Development. Denver test. Day care centers. Food supplement.

\section{REFERÊNCIAS BIBLIOGRÁFICAS}

1. Oria H. Creches: perspectivas atuais. Rev Paul Pediatr 1988; 6 (23): 141-48.

2. Organizacion Mundial de la Salud. Problemas de atención de la salud del niño de corta edad en un contexto ecológico en desarrollo. Bol Sanit Panamer 1980; 89(3): 249-62.

3. Valente J BS. Fome e desnutrição: determinantes sociais. São Paulo, Ed. Cortez, 1986.
4. Alvim EF, Costa RA. Avaliação do estado nutricional das crianças menores de cinco anos, nas áreas de atuação da FSESP. Rev FSESP 1979; 24 (1): 32-49.

5. Aukett MA. Parks YA et al. Treatment with iron increases seight and psychomotor development. Arch Dis Child 1986; 61: 849-57.

6. Nóbrega FJ . Desnutrição intra-uterina e pós-natal. São Paulo, Ed. Panamed., 2 $2^{\mathrm{a}}$ ed, 1986.

7. Brasil. Ministério da Saúde. Instalação e funcionamento de creches. Normas e Manuais Técnicos. Brasília, 1986, mímeo.

8. Conselho Nacional dos Direitos da Mulher (CNDM). Organização e funcionamento da creche. Brasília, 1988.

9. Oliveira ZMR, Ferreira MCR. Propostas para o atendimento em creches no município de São Paulo. Cadernos de Pesquisa 1986; 56: 39-65.

10. Prugh GD, Harlow GR. Privación encubierta en lactantes y niños pequenos. Cadernos de Salud Publica. Organización Mundial de la Salud. Ginebra, 1963.

11. Frankenbur G, Dodds. The Denver Developmental Screening Test. J Pediatr 1971; 71: 181.

12. Landis J R, Koch GG. The measurement of observer agrement for categorical data. Biometr 1977; 33: 159-74.

13. Remington RD, Schork MA. Statistics with aplications to the biological and Health Sciences. Prentice-Hall, Inc., Englewood. Cliffs New J ersey, 1970. 